\title{
Germanica
}

\section{SpielGeldSpiel - Der Spekulant als Reflektionsfigur in der deutschen Gegenwartsprosa}

Jonas Luscher : Frühling der Barbaren, 2013

Daniel Kehlmann : F, 2013

Sasha Reh : Gibraltar, 2013

Le jeu des enjeux - Le spéculateur en personnage réflexif dans la prose

allemande contemporaine

Playing money games - The speculator as a reflection character in contemporary

German prose

Jonas Nesselhauf

\section{(2) OpenEdition}

Journals

Édition électronique

URL : http://journals.openedition.org/germanica/2665

DOI : 10.4000/germanica.2665

ISSN : 2107-0784

\section{Éditeur}

Université de Lille

\section{Édition imprimée}

Date de publication : 30 décembre 2014

Pagination : 81-96

ISBN : 9782913857346

ISSN : 0984-2632

\section{Référence électronique}

Jonas Nesselhauf, « SpielGeldSpiel - Der Spekulant als Reflektionsfigur in der deutschen

Gegenwartsprosa », Germanica [Online], 55 | 2014, Online erschienen am: 30 Dezember 2016,

abgerufen am 06 Oktober 2020. URL : http://journals.openedition.org/germanica/2665 ; DOI : https:// doi.org/10.4000/germanica.2665 


\title{
SpielGeldSpiel - Der Spekulant als Reflektionsfigur in der deutschen Gegenwartsprosa
}

\author{
Jonas Luscher : Frühling der Barbaren, 2013 \\ Daniel Kehlmann : F, 2013 \\ Sasha Reh : Gibraltar, 2013
}

Jonas NESSELHAUF

Vechta / Saarbrücken

Die globale Wirtschafts- und Finanzkrise der Jahre 2007 und 2008, die mit ihren Folgeerscheinungen zum steten Begleiter der darauf folgenden Jahre wurde, produzierte, wie kaum ein anderes Ereignis des frühen 21. Jahrhunderts, allgemeine und individuelle Ängste und Unsicherheiten. Und nicht zuletzt die Auswirkungen auf den europäischen Arbeitsmarkt und Handel, wie auch die jeweilige Binnenwirtschaft und Staatsverschuldung, führten zu einem tiefen Misstrauen in Politik und Ökonomie.

In den Blickpunkt geriet dabei immer wieder das Bankensystem, dem eine ungezügelte und vor allem unkontrollierte Gier vorgeworfen wurde. So ging die Höhe der jährlich ausgeschütteten Bonuszahlungen mancher Finanzdienstleister ebenso durch die Presse wie Skandalmeldungen ausschweifender Partys und Luxusreisen der Vorstände oder die ungeheuer anmutenden Summen innerhalb nur weniger Wochen verzockter Anlagen. Diese scheinbar außer Kontrolle geratene ,Schattenwirtschaft' des Turbokapitalismus wurde schnell zum Mitschuldigen der globalen Finanzkrise erklärt, Fälle wie AIG oder Lehman Brothers auf mensch- 
liche Gier und ein kaum überwachtes riskantes Spiel mit dem Geld zurückgeführt.

Die Manifestation dieses Phänomens findet sich in Gestalt des Spekulanten, der ohnehin eine Ausnahmeerscheinung und Randfigur innerhalb des ökonomischen Systems darstellt ${ }^{1}$. Schließlich, so der Vorwurf seit jeher, leiste er keine Arbeit im herkömmlichen Sinne, sondern lasse vielmehr das (meist fremde) Geld arbeiten, während die Spekulation selbst eher dem Glücksspiel gleiche, ein „,,Rückfall in die Barbarei'“2 darstelle und den von Max Weber aufgestellten kapitalistischen Grundsatz der „Berechenbarkeit“3 verletze. Während der (klassische) Kaufmann gezielt und umsichtig Handeln und Investieren müsse, erziele der Spekulant dagegen nahezu „ohne Gegenleistung unermessliche Gewinne" ${ }^{\text {" }}$. Dadurch und aufgrund der immer höheren Summen, mit denen hantiert werde, sei zudem längst ,jeder Bezug zur Realwirtschaft [...] verloren gegangen"5, was wiederum zu einer regelrechten Maßlosigkeit führe 6 . Geld existiere nur noch als bloße Zahlen, der Handel werde zum Spiel, die anvertrauten Investitionen zum ,Spielgeld'.

Doch - zur Rettung dieser meist männlichen Figur: Bereits in frühen theoretischen Auseinandersetzungen wird immer wieder auf die Kreativität und (spekulative) Phantasie hingewiesen, die der Spekulant für seine Arbeit benötigt: „Er muss in den gegenwärtigen Marktentwicklungen sehen, was andere (noch) nicht sehen können"7. Und das selbst, obwohl ein Großteil des Investment- und Börsenhandels heute, der Echtzeit angenähert, tatsächlich von Algorithmen und Computerprogrammen getätigt wird: Hedgefonds oder Aktienoptionen sind etwa so programmiert, dass bei Erreichen eines gewissen Wertes automatisch ge- oder verkauft wird. Und nicht nur in Robert Harris Thriller The Fear Index (2011) wird ein Algorithmus zur Bedrohung,

1. - Vgl. Urs Stäheli, „Der Spekulant“, In: Stephan Moebius und Markus Schroer (Hrsg.): Diven, Hacker, Spekulanten. Sozialfiguren der Gegenwart, Frankfurt am Main, Suhrkamp, 2010. S. 353-365. Wenn in diesem Aufsatz vom ,Spekulanten' gesprochen wird, geschieht dies oftmals verallgemeinernd und schließt natürlich andere Finanzdienstleister wie Investmentbanker etc. mit ein.

2. - Heinz-Dieter Assmann und Karl-Josef Kuschel, Börsen, Banken, Spekulanten. Spiegelungen in der Literatur - Konsequenzen für Ethos, Wirtschaft und Recht, Gütersloh, Gütersloher Verlagshaus, 2011. S. 121.

3. - Vgl. Jürgen Kaube, „Arbeit, Verschuldung, Spekulation. Drei Zumutungen des Kapitalismus.“ In: Neue Rundschau 1 (2001). S. 18.

4. - Urs Stäheli, ,Der Spekulant“, a.a.O. S. 354.

5. - Heinz-Dieter Assmann und Karl-Josef Kuschel, Börsen, Banken, Spekulanten, a.a.O. S. 121 .

6. - Vgl. Christina von Braun, Der Preis des Geldes. Eine Kulturgeschichte, Berlin, Aufbau, 2012. S. 73.

7. - Urs Stäheli, ,Der Spekulant“, a.a.O. S. 355. 
entpuppt sich im Roman als programmiertes Monster, das zwar weiter Gewinne einfährt, aber längst außer Kontrolle geraten ist. Denn tatsächlich verzeichneten Finanzaufsichtsbehörden bereits mehrfach einen sogenannten „flat crash“, bei dem Computerprogramme automatisch auf kurzzeitige Verkaufswellen reagieren und (natürlich unreflektiert) nachziehen ${ }^{8}$.

Daher überrascht es kaum, dass die Figur des Spekulanten in der Literatur der vergangenen Jahre auch zu einer Metapher für den Turbokapitalismus, die Marktwirtschaft und pars pro toto auch für das gesamte kapitalistische Wirtschaftssystem wurde. An ihm lassen sich unterschiedliche Auffassungen von Arbeit und Handel aufzeigen, moralische Fragen und Probleme durchspielen; als Symbol für Gier und Maßlosigkeit stellt er einen Gegensatz zum klassischen Arbeiter und ein Hassobjekt der ,Occupy'-Bewegung dar.

So arbeitet sich vor allem die britische Literatur mit dem einschlägigen Finanzplatz London an dieser (wieder aktuell gewordenen) interessanten und mehrschichtigen Sozialfigur des frühen 21. Jahrhunderts ab - etwa in John Lanchesters Roman Capital (2012) oder Sebastian Faulks multiperspektivischem A Week In December (2009), indirekt auch in Zadie Smiths NW (2012) und dem bereits erwähnten Fear Index.

Und auch in deutschsprachigen Bearbeitungen der Weltwirtschaftskrise findet eine Auseinandersetzung mit der Figur des Spekulanten statt - nach einer kurzen literaturgeschichtlichen Skizze sollen dafür exemplarisch Werke von Jonas Lüscher, Daniel Kehlmann, Sascha Reh und Kathrin Röggla herangezogen werden, unterstützt durch ausgewählte Texte von Nora Bossong, Elfriede Jelinek und Andres Veiel.

\section{Eine kurze Literaturgeschichte des Spekulanten}

Die Figur des Spekulanten wurde von der Literatur verhältnismäßig spät entdeckt, auch weil sie lange Zeit als eher, langweilig' galt. Dies ändert sich in der Mitte des 19. Jahrhunderts - und auch sicher als Folge zeitaktueller Wirtschafts- und Finanzkrisen ${ }^{9}-$, als die tatsächliche Gefahr von riskanten Spekulationen, gleichzeitig aber auch deren literarisches Potential erkannt wird: „Gerade der moralische Fall, Täuschung und der schnelle Wechsel des Schicksals in spekulativen Manien wecken das Interesse des Lesers."10

8. - Vgl. Michaela Schiessl, „Missratene Kreatur.“ In: Der Spiegel 45 (2011). S. 96.

9. - Ein gut recherchierter geschichtlicher Überblick über Finanz- und Wirtschaftskrisen, der diesem Aufsatz zugrunde liegt, findet sich bei: Robert Aliber und Charles Kindleberger, Manias, Panics and Crashes. A History of Financial Crises, New York, Palgrave Macmillan, 2011.

10. - Urs Stäheli, Spektakuläre Spekulation: Das Populäre der Ökonomie, 
Anlehnungen an tatsächliche Fälle (wie dem Crash der Banque de l'Union Générale im Jahre 188211), finden sich etwa auch innerhalb von Émile Zolas Zyklus Les Rougon-Macquart und konkret am Beispiel von Aristide Saccard aus L'Argent (1891), der sich mit seiner selbst gegründeten Banque Universelle folgenreich verspekuliert. Bereits hier ist und war der Börsenhandel ein risikoreiches Unterfangen - Erfolg und Misserfolg, Aufstieg und Untergang, Gewinnen und Zerrinnen liegen nah beieinander: „Les grosses sommes aussi vite perdues que gagnées"12. Paradigmatisch dafür steht Zolas Milieuschilderung des geschäftigen Treibens rund um den Place de la bourse, schließlich herrscht an der Börse für den Anleger immer ,Krise', selbst wenn seine Aktien gut stehen: ,[Le] grand argument contre le jeu était que, mathématiquement, le joueur devait toujours perdre: s'il gagne, il a à déduire le courtage et le droit de timbre; s'il perd, il a en plus à payer les mêmes droits $[\ldots]$.. 13 .

Und auch nach unzähligen Wirtschaftskrisen hat sich an dieser ,mathematischen Gewissheit' des sicheren Verlustes an der Börse nichts geändert - der Spekulant befindet sich, wie bereits Zolas Zeitgenosse Heinrich Heine in seinen essayistischen Artikeln Französische Zustände im Mai 1832 beobachtet, metaphorisch auf einem Schiff in turbulenten Gewässern, das sich ,,[...] wogend und brausend [...] bewegt, wie ein Meer des Eigennutzes, wo aus den wüsten Menschenwellen die großen Bankiers gleich Haifischen hervorschnappen, wo ein Ungetüm das andere verschlingt, und wo oben auf der Galerie, gleich lauernden Raubvögeln auf einer Meerklippe, sogar spekulierende Damen bemerkbar sind" 14 .

Während Heine - in seinem ohnehin sehr gespaltenen Verhältnis zu Geld und Kapitalismus - den Spekulanten als „Staatspapierenschacher“15 beschimpft, sieht Karl Marx etwa zeitgleich in dieser ökonomischen Ausprägung eine Gefahr für die Gesellschaft generell: „Die Geldgier oder Bereicherungssucht ist notwendig der Untergang der alten Gemeinwesen"16.

Denn risikoreiche Spekulationen gefährden nicht nur die öffentliche Ordnung, sondern reißen auch oftmals arglose Sparer mit in den Abgrund, vom Versprechen des schnellen Geldes angelockt. So

Frankfurt, Suhrkamp, 2007. S. 9.

11. - Vgl. Sandra Richter, Mensch und Markt. Warum wir den Wettbewerb fürchten und ihn trotzdem brauchen, Hamburg, Murmann, 2012. S. 123.

12. - Émile Zola, L'Argent, Paris, Gallimard, 2009. S. 21.

13. - Ebd. S. 234.

14. - Heinrich Heine, Sämtliche Werke Band III, Schriften zur Literatur und Politik I., Darmstadt, Wissenschaftliche Buchgesellschaft, 1992. S. 166.

15. - Etwa ebd. S. 165f.

16. - Karl Marx, „Grundrisse der Kritik der politischen Ökonomie.“ In: Ders.: Ökonomische Schriften 1857/58, Berlin, Dietz, 1983. S. 149. 
zeigen bereits frühe Romane über Börsenkrisen - neben Émile Zolas L'Argent etwa auch Frank Norris The Pit (1903) oder Upton Sinclairs The Money Changers (1908) ${ }^{17}$ - die Hilflosigkeit der Kleinanleger, die das System der Aktienspekulationen nicht völlig durchschauen. Wenn ihre Ersparnisse und teilweise ihr gesamtes Vermögen dann innerhalb weniger Augenblicke zusammengeschrumpft ist, berufen sie sich entschuldigend auf das Individuum, das Spieltrieb und Risikolust „démence du jeu“18 - verfallen sei: „Il y a, dans la passion du jeu, un ferment désorganisateur que j’ai observé souvent, qui ronge et pourrit tout, qui fait de la créature de race la mieux élevée et la plus fière une loque humaine, le déchet balayé au ruisseau..."19

Selbst mit der aufkommenden Massenpresse kann der ,einfache Mann' aus dem Volk den immer komplexer werdenden Diskurs nicht durchblicken und sieht weiterhin das triebgesteuerte Individuum, den gierigen Großkapitalisten, als Verursacher der Krise. Emma Pinneberg etwa konstatiert in Hans Falladas Roman Kleiner Mann - was nun? (1932): „Wie kann man lachen, richtig lachen, in solcher Welt mit sanierten Wirtschaftsführern, die tausend Fehler gemacht haben, und kleinen entwürdigten, zertretenen Leuten, die stets ihr Bestes taten?"20 Und in Ödön von Horváths Stück Kasimir und Karoline (1932) ruft der gerade entlassene Kasimir beim Anblick eines Zeppelins aus: „Da fliegen droben zwanzig Wirtschaftskapitäne und herunten verhungern derweil einige Millionen!" 21

Dennoch spielt die Figur des Spekulanten in der literarischen Beund Verarbeitung der Weltwirtschaftskrise von 1929 insgesamt eine eher untergeordnete Rolle. Und auch die Erkenntnis, dass Krisen eine unvermeidbare Bedingung des wirtschaftlichen Systems darstellen, entwickelt sich in dieser Form erst nach dem verheerenden Einschnitt vom Oktober 192922.

Eine umso bedeutendere Rolle kommt dem Spekulanten dafür nach der aktuellen Krise von 2007ff. zu, brachte doch die (öffentlich wahrgenommene) Gier nach Profit und Bonuszahlungen eine ganze Berufsgruppe in Misskredit. Zu den bekanntesten Fällen zählen die riskanten Spekulationsgeschäfte von Jérôme Kerviel, der sich mit der

17. - Vgl. zu diesen Texten einführend: Zimmerman, David: Panic! Markets, Crises, and Crowds in American Fiction, Chapel Hill, University of North Carolina Press, 2006.

18. - Zola, L'Argent. a.a.O. S. 417.

19. - Ebd. S. 436.

20. - Hans Fallada, Kleiner Mann - was nun?, Berlin, Aufbau, 2012. S. 334.

21. - Ödön von Horváth, Kasimir und Karoline, Frankfurt am Main, Suhrkamp, 2009. S. 12.

22. - Vgl. dazu etwa Werner Plumpe, Wirtschaftskrisen. Geschichte und Gegenwart, München, C.H. Beck, 2012. S. 8f. 
Société Générale um fast fünf Milliarden Euro verzockte, während Kweku Adoboli seinem Arbeitgeber, der UBS, mehr als zwei Milliarden Dollar Schaden zufügte. Andere Banken und Finanzdienstleister mussten ähnliche Fälle eingestehen, etwa JP Morgan Chase im Frühjahr 2012.

Nun kann längst nicht mehr nur die Hybris des Individuums (wie noch bei Aristide Saccard) für solch enorme Fehlspekulationen verantwortlich gemacht werden, sondern nicht zuletzt das System selbst, das dies ohne nennenswerte Schranken und Kontrollen zulässt, vielleicht sogar begünstigt. In einer überarbeiteten Rede, die Slavoj Žižek vor Occupy-Aktivisten am Zuccotti Park hielt, fasst der Philosoph zusammen: „Schiebt die Schuld nicht auf die Einstellungen der Menschen. Nicht Korruption und Habgier sind das Problem, sondern

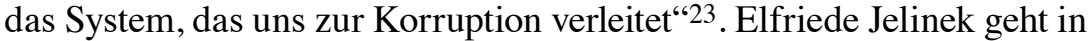
ihren kapitalismuskritischen Texten wie Die Kontrakte des Kaufmanns (2009) oder Rein Gold (2013) noch einen Schritt weiter: Das System erlaubt es dem Spekulanten nicht nur, es provoziert regelrecht ein besonders risikoreiches Anlageverhalten durch fehlende Regulationen und Kontrollinstanzen:

Der Mensch denkt, doch Gott lenkt, aber uns lenkt er nicht, wir lenken selbst, wir lenken die Firmen, die heißen wie wir [...], Sie glauben es immer noch nicht, eine Plausibilisierung durch die Prüfer hat zwar ergeben, daß wir Lizenzgebühren in der Höhe von höchstens, das ist hoch die Höhe!, nicht wahr, einer Million Euro hätten abrechnen dürfen, doch wer verlangt von uns, dass wir rechnen können müssen? ${ }^{24}$

Ein weiterer Schwerpunkt in literarischen Texten der aktuellen Wirtschaftskrise liegt in der Verknüpfung der verschiedenen ökonomischen Beziehungen, was wiederum auf die Chronologie der Krise zurückzuführen ist. Als Reaktion auf die verheerenden Folgen der „Dotcom-Spekulationsblase“ 25 wurden Zinsen über Jahre hinweg niedrig gehalten, was vor allem in den USA, sowie in Spanien und Irland zu einem Immobilienboom führte. Als diese Blase wiederum im Sommer 2007 platzte, kam es zu weitläufigen Zahlungsausfällen, was Banken und Finanzdienstleister in Bedrängnis brachte - den Höhepunkt

23. - Slavoj Žižek: „Das gewaltsame Schweigen eines Neubeginns.“ In: Carla Blumenkranz et al.: Occupy! Die ersten Wochen in New York. Eine Dokumentation, Frankfurt am Main, Suhrkamp, 2011. S. 68.

24. - Elfriede Jelinek, Die Kontrakte des Kaufmanns, Reinbek, Rowohlt, 2009. S. 252.

25. - Besonders die Aktien von Technologiekonzernen erlebten Mitte der 1990er Jahre unter dem Namen „Neuer Markt“ einen regelrechten Boom an den Börsen. Vgl. dazu und dem folgenden Abriss überblickend: Robert Aliber und Charles Kindleberger, Manias, Panics and Crashes. a.a.O. S. $180 \mathrm{ff}$. 
stellten zweifellos die Absicherung der US-amerikanischen AIG mit mehreren hundert Milliarden Dollar Staatshilfe, sowie die Pleite von Lehman Brothers im September 2008 dar. Schnell zeigen sich auch Auswirkungen auf die Realwirtschaft: Als Produktivität und Kaufkraft einbrechen, kommt es fast überall auf dem Globus zu einer Rezession, in deren Verlauf schließlich zu einer Staatsschuldenkrise, die vor allem Griechenland an den Rande des Staatsbankrotts führte. Die vielfachen und miteinander verknüpften Folgen rufen erneut ins Bewusstsein zurück, wie stark unterschiedlichste Bereiche des alltäglichen Lebens über ökonomische Beziehungen miteinander verbunden sind ${ }^{26}$.

Insgesamt, so lässt sich diese kurze Stoff- und Motivgeschichte der Figur des Spekulanten abschließen, liegt sein Reiz in der Darstellung einer (beispielhaften) Einzelgeschichte, damit verbunden aber auch in der Kontrastierung unterschiedlicher ökonomischer Mentalitäten (Max Weber). Dies führt oftmals zu moralischen Fragen oder gar Dilemmata, zentral natürlich aber zur Gegenüberstellung von Kapitalismusgewinnern und Krisenverlierern.

Um diese aktuelle Tendenz in der deutschsprachigen Prosaliteratur aufzuzeigen, werden exemplarisch vier literarische Werke einer Generation junger Schriftsteller herangezogen. Dabei sollen anhand von Jonas Lüschers Novelle Frühling der Barbaren (2013) und Daniel Kehlmanns Roman $F$ (2013) die Figur des Spekulanten inhaltlich untersucht werden, während an Sascha Rehs Gibraltar (2013) und Katrin Rögglas wir schlafen nicht (2004) eher formale und narrative Strategien der Darstellung im Mittelpunkt stehen werden.

\section{Der Habitus des Spekulanten - Frühling der Barbaren}

Die Novelle Frühling der Barbaren des Schweizer Schriftstellers Jonas Lüscher erzählt vom britischen Staatsbankrott, den der Geschäftsmann Preising fernab in einem Urlaubsresort in der tunesischen Wüste erlebt. Neben dem Besitzer des international agierenden Prixxing-Unternehmens befindet sich dort - ausgerechnet im Thousand and One Night Resort 27 in der Oase Tschub - auch eine dekadente Londoner Hochzeitsgesellschaft. Die vorwiegend aus Bankern und Geschäftsleuten bestehende Gruppe bereitet eine luxuriöse Trauung vor, wird dann aber vom Zusammenbruch der heimischen Wirtschaft überrascht. Zunehmend lädt sich die Situation auf, nachdem das Britische Pfund schnell an Wert verliert und die Kreditkarten der Gäste gesperrt

26. - Vgl. John Lanchester, I.O.U. Why Everyone Owes Everyone and No One Can Pay, New York, Simon \& Schuster, 2011. S. 6.

27. - Jonas Lüscher, Frühling der Barbaren, München, C.H. Beck, 2013. S. 29. Kursivierung übernommen. 
werden. Die Raserei gipfelt schließlich in der totalen Zerstörung der Hotelanlage und einer regelrechten Hetzjagd auf Tiere und Angestellte. In letzter Minute kann Preising dem wütenden Mob nach Tunis entkommen, doch längst haben die britischen Urlauber alle Grundsätze der Zivilisation und des Zusammenlebens abgelegt und brennen das Wüstenresort sogar nieder.

In der klassischen Novellenform aus Rahmen- und Binnenerzählung fungiert Preising als (meist neutraler) Außenstehender, der die verschiedenen Stufen der Eskalation hautnah miterlebt, und auch bereits zuvor den gruppendynamischen Habitus der Spekulanten kritisch beobachtete.

Junge Leute in ihren späten Zwanzigern und frühen Dreißigern. Laut und selbstsicher. Schlank und durchtrainiert. [...] Selbst nahezu nackt wirkten sie wie in Uniform. [...] Sie standen Witze reißend an einer der Bars, sie verschwanden, sich ungestüm küssend und sich die Hände gegenseitig unter die Bünde ihrer engen Shorts steckend, in ihren klimatisierten Zelten, sie erteilten dem Personal selbstsicher Anweisungen, sie wanderten fluchend durch die Palmenhaine auf der Suche nach besserem Empfang für ihre Blackberrys [... $]^{28}$.

Ihr Erscheinen folgt dabei - selbst im Urlaub und außerhalb der Londoner City - systemeigenen Verhaltensweisen und Männlichkeitskonstruktionen, die an den Habitus der New EconomyGeneration erinnern ${ }^{29}$. Auftreten und ein festgelegter Markenkodex gehören zum Selbstbild wie Besitz und Luxus zum Selbstverständnis dieser jungen Generation von Bankern, die einen quasi normativ vorgeschriebenen Code zu befolgen scheint.

Dieses Phänomen findet sich auch in anderen literarischen Bearbeitungen der rezenten Wirtschaftskrise, exemplarisch etwa in John Lanchesters Roman Capital und dort am Beispiel des Investmentbankers Roger Yount. Obwohl er selbst kein besonderes Interesse an der Jagd oder an Wohltätigkeitsbällen verspürt, ist dies unbedingter Teil der Gruppenzugehörigkeit: „He even sponsored charities, not out of charitable feeling [...] of any kind, not for anybody - but because it was what you did if you were that rich. It was as if there was a rule book" 30 .

So gelingt es bereits durch diesen Einzelaspekt typischer Verhaltensmuster, das Soziogramm einer jungen Spekulantenriege zu zeichnen; allein durch Beschreibungen ihrer Mentalität und ihres

28. - Ebd. S. 33f.

29. - Vgl. Jonas Nesselhauf, „Geld. Macht. Potenz. - Inszenierungen und Repräsentationen männlicher Bankrotteure in literarischen Bearbeitungen der Weltwirtschaftskrise." <https://www.fk12.tu-dortmund.de/cms/ISO/de/soziologie/ soziologie_der_geschlechterverhaeltnisse/Medienpool/AIM_2013_Tagung/Nesselhauf_ Inszenierung_und_Repr_sentationen_maennlicher_Bankrotteure.pdf $>$.

30. - John Lanchester, Capital, London, Faber and Faber, 2013. S. 96. 
Auftretens wird innerhalb des Textes eine (passive) Charakterisierung vorgenommen. Bei Jonas Lüscher spielt darüber hinaus auch der Raum eine nicht unbedeutende Rolle: Das Resort in der tunesischen Wüste ist nicht nur Ort der opulenten Hochzeit, sondern auch eine Chiffre für den durchkapitalisierten und - trotz der Lage in Nordafrika - nach westlichen Standards ausgerichteten Tourismus des globalisierten 21. Jahrhunderts. Gleichzeitig wird der abgeschlossene Hotelkomplex aber auch zu einem Experimentalraum, durchaus im Sinne des Naturalismus, schließlich stellt der Staatsbankrott Englands nicht nur (novellentheoretisch) den Wendepunkt und die ,unerhörte Begebenheit' dar - „Während Preising schlief, ging England unter" 31 -, sondern auch den (wortwörtlichen) Offenbarungsmoment der britischen Banker. Denn der ökonomische Konkurs der heimischen Volkswirtschaft geht symbolisch mit einer moralischen Bankrotterklärung dieser Generation besonders aggressiver Spekulanten einher: Nachdem die Kreditkarten gesperrt wurden und das Bier im Resort ausgeht, verwüsten sie die Anlage, schlachten Tiere $a b$ und lassen einen Hotelangestellten im Pool ertrinken ${ }^{32}$.

Und jetzt erscheint das Verhalten dieser kolonialistisch und skrupellos agierenden Banker als ,barbarisch', es kommt tatsächlich zum titelgebenden Frühling der Barbaren ${ }^{33}$. Aber erst jetzt? Denn, so eine mögliche Interpretation, schließlich reagieren die Spekulanten ja nur mit den gleichen Verhaltensweisen auf die Krise im Wüstenresort, wie sie ohnehin arbeiten, nämlich selbstbewusst und aggressiv ${ }^{34}$. Und nicht zuletzt wurde das riskante Spiel mit Geld ja ohnehin immer wieder von Kritikern, wie zu Beginn angeführt, als ,Rückfall in die Barbarei' bezeichnet.

So offenbart der Zusammenbruch der Ordnung infolge einer ökonomischen Pleite nun auch den moralischen Bankrott dieser jungen Generation von Spekulanten, die zwar wie selbstverständlich mit dem Kapitalismus aufgewachsen ist und dessen Denkmuster verinnerlicht hat, nun aber eben während einer Krise auch nur mit den Strategien eines aggressiven Turbokapitalismus reagieren kann.

31. - Lüscher, Frühling der Barbaren. a.a.O. S. 89. Die Staatspleite selbst wurde zu einem wahrlich ikonischen Moment, der sich im kollektiven Gedächtnis festsetzte (Vgl. ebd. S. 96).

32. - Vgl. ebd. S. 114-120.

33. - Auch bereits im der Novelle paratextuell vorangestellten kurzen Text des Historikers Franz Borkenau, der ,Barbarei' als einen Zustand ohne „gesellschaftliche und moralische Kohärenz" (ebd. S. 5) definiert.

34. - Vgl. Nesselhauf, „Geld. Macht. Potenz.“ a.a.O. S. 6f. und 9f. 


\section{Die Regeln des Spiels - F}

Daniel Kehlmanns Roman $F$ widmet sich episodisch (und an Einzelereignissen sogar multiperspektivisch) drei sehr unterschiedlichen Geschwistern: Eric Friedland arbeitet als Finanzberater und Investmentbanker, sein Bruder Iwan als Künstler und vor allem Kunstfälscher, während ihr Halbbruder Martin ein katholischer Geistlicher ist. Geschickt verwoben finden die unterschiedlichen Erzählstränge immer wieder lose zueinander; darüber hinaus erinnern einzelne Motive leicht an Kehlmanns früheren Roman Beerholms Vorstellung (1997), etwa in Bezug auf Figuren wie auch Motive. So kommt dem Thema des Schicksals - und ,Fatum' wäre tatsächlich eine von mehreren Deutungsmöglichkeiten für den kryptischen Romantitel - auch für den Spekulanten Eric eine nicht unbedeutende Rolle zu, schließlich hat er bereits vor der eigentlichen Weltfinanzkrise das Vermögen seines wichtigsten Kunden verzockt - sein Eingeständnis wird zur nüchternen Bankrotterklärung: „Ich verwalte das gesamte Vermögen von Adolf Alfred Klüssen, und ich habe alles verloren“ 35 .

Um den Schein aufrecht zu erhalten, erfindet er Bilanzen und weist falsche Gewinne aus - ständig benötigt Eric das Geld neuer Kunden, „um die Rendite der alten Anleger zu bezahlen“36, ein Teufelskreis, den erst die tatsächliche Weltwirtschaftskrise beendet. Zwar wurde Erics Vermögen gepfändet und seine Ehe geschieden, doch Klüssen und andere Anleger akzeptieren nun (unwissentlich und unter dem Deckmantel der Krise) ihre eigentlich schon viel früher erlittenen Verluste ${ }^{37}$.

Dennoch ist Eric ein nicht untypischer Krisengewinner, der wie viele andere Spekulanten seinen eigenen Bankrott relativ glimpflich erlebt und sogleich ein neues Jobangebot erhält - man denke etwa nur an den Pariser Spekulationskönig Aristide Saccard, der in Zolas L'Argent ein neues Leben im Ausland beginnen kann, während seine Opfer mittellos und finanziell ruiniert zurückbleiben. Er ist auch ein typischer Spekulant, dessen Mentalität und Verhaltensweisen an die britischen Banker zuvor anschließt. Denn erneut stellt nicht nur das capital économique (die Geldvermehrung durch Spekulation) eine treibende Kraft für den Banker dar, sondern auch - Pierre Bourdieus Konzept folgend - das capital culturel, zu dem ja schließlich auch „klassenspezifische Kenntnisse und Eigenschaften" 38 gehören. Daher richtet sich Erics Außendarstellung (zusätzlich die Selbstwahrnehmung bedingend) nach dem systemintern vorgegebenen Habitus, etwa wenn er sagt: „Ein Mann

35. - Daniel Kehlmann, $F$, Reinbek, Rowohlt, 2013. S. 182.

36. - Ebd. S. 188.

37. - Vgl. ebd. S. 375.

38. - Vgl. Braun, Der Preis des Geldes. a.a.O. S. 182. 
in meiner Position muss nun mal ein sehr teures Gemälde besitzen“39. Und so befinden sich in seinem Arbeitszimmer nicht nur ungelesene Bücher, sondern auch ein Originalgemälde von Paul Klee; das Haus der Familie ist darüber hinaus mit einem hochwertigen Medienraum ausgestattet, während Eric - in luxuriösen Maßanzügen - über einen persönlichen Chauffeur verfügt.

Umgekehrt zeichnet er sich aber auch dadurch als (offensichtlich typischer) Banker aus, da er seine tägliche Arbeit nur unter dem Einfluss von Medikamenten ausüben kann - durchaus dem zeitaktuellen Diskurs des literarischen Spekulanten entsprechend und etwa auch in Sascha Rehs Gibraltar zu finden. Mehrmals am Tag muss Eric verschiedenste Beruhigungsmittel einnehmen, so dass Beobachtung wie auch Eigenperspektive stark eingeschränkt sind. Diese getrübte Wahrnehmung wird auch narrativ in der (allerdings nicht durchgehend) autodiegetischen Erzählung von Eric gespiegelt, die durch Abschweifungen, Fragmentierung und Verwirrtheit geprägt ist. Andere Figuren, das zeigen ihre Reaktionen oder Äußerungen in späteren Kapiteln, empfinden sein Verhalten als zerstreut und geistig abwesend; Eric selbst nimmt sein Umfeld kaum wahr, halluziniert und leidet unter Verfolgungswahn.

Dieser Realitätsverlust hat auch zwangsläufig Folgen auf sein Arbeitsethos - Eric setzt trotz verheerender Fehlspekulationen weiter darauf, den ewigen Kreis, alte Rendite mit dem Kapital neuer Anleger zu bezahlen, fortzuführen. Und das, obwohl er sich bewusst ist, dass sein Spiel zwangsläufig zu einem Ende kommen wird und die gefälschten Bilanzen auffliegen werden. Und ironischerweise ist es nun ausgerechnet die überraschend einsetzende Wirtschaftskrise, die Erics bereits längst zuvor eingefahrene Verluste abmildert, seinem Spiel ein (aus seiner Sicht) noch glimpfliches Ende bereitet.

\section{Multiperspektivisches Krisenerzählen - Gibraltar}

Auch Sascha Rehs Gibraltar kreist um eine verhängnisvolle Fehlspekulation und deren Folgen: Hier ist es Bernhard Milbrandt, der eine traditionsreiche Privatbank durch den Handel mit griechischen Staatsanleihen an den Rande der Insolvenz führt. Als die Verluste zu hoch werden, setzt er sich ab und taucht in einer Planstadt unter, die an der spanischen Küste während des Immobilienbooms errichtet wurde, seit dem Platzen der Spekulationsblase und Beginn der Finanzkrise aber unbewohnt einer unwirklichen Ruinenlandschaft gleicht.

Interessant ist nun, dass der Roman diese Geschichte nicht stringent und durch eine gleich bleibende (vielleicht heterodiegetische und

39. - Kehlmann, F. a.a.O. S. 164. 
dadurch auch kommentierende) narrative Instanz erzählt. Vielmehr werden multiperspektivisch die Blickwinkel von zentralen Figuren gleich einer Collage zusammengebracht: Gerahmt von jeweils einer (neutralen) Zeitungsmeldung kommen die sechs Hauptbeteiligen zu Wort - und erst im Abgleich aller Perspektiven entsteht das Gesamtbild. So liegt der Fokus etwa im dritten Kapitel in zwei ineinanderfließenden Erzählsträngen auf dem Spekulanten Bernhardt Milbrandt, während der inzwischen verstorbene Bankenpatriarch Johann Alberts im darauffolgenden vierten Kapitel autodiegetisch selbst berichtet.

Das multiperspektivische Erzählen - in der deutschen Literaturwissenschaft noch immer theoretisch sehr unscharf ${ }^{40}$ - ist häufig auf Kontrastierung ausgelegt; dabei können beispielsweise verschiedene Meinungen, Einstellungen und Mentalitäten einander gegenüber gestellt werden, um Unterschiede zwischen Generationen oder im Arbeitsethos aufzudecken. Sicherlich findet sich diese Strategie ansatzweise bereits auch in Lüschers Frühling der Barbaren wieder, wenn das Verhalten der jungen Generation dem der älteren Banker und Spekulanten gegenübergestellt wird ${ }^{41}$, dort aber nur innerhalb der Erzählung und lediglich durch Preising oder den Rahmenerzähler der Novelle wiedergegeben oder kommentiert. Deutlich multiperspektivischer arbeitet Kehlmanns Roman $F$, in dem das Einzelereignis eines Mittagessens zwischen dem Spekulanten Eric und seinem Bruder Martin gleich zwei Mal aus unterschiedlichen Blickwinkeln wiedergegeben wird ${ }^{42}$. Dabei erscheint Erics für den Geistlichen peinliche Frage nach Horoskopen ebenso wie Erics ständige Blicke auf das Handy aus zwei verschiedenen Perspektiven, und auch Martins Verwunderung über das konfuse und teils abwesende Verhalten seines Bruders wird durch den zweiten Blickwinkel an späterer Stelle für den Rezipienten erklärbar.

Eine noch stärkere Synthetisierungsleistung des Lesers fordert Sascha Rehs Gibraltar, in der Tradition der multiperspektivischen Detektivgeschichte stehend. Die sechs aneinander gefügten Perspektiven ergeben das Gesamtbild einer fatalen Fehlspekulation und ihrer Folgen für die beteiligten Personen wie auch die Privatbank Alberts. Diese ungewöhnliche narrative Form ermöglicht nicht nur einen durchkomponierten Spannungsaufbau durch die Anordnung der einzelnen

40. - Neben einem Eintrag im Metzler Lexikon Literatur- und Kulturtheorie (Stuttgart 2008) sind lediglich zwei Standardwerke zu nennen, die sich dezidiert mit dem Phänomen der Multiperspektivität auseinandersetzen: Die Untersuchung Typen multiperspektivischen Erzählens von Volker Neuhaus (Köln 1971) und der von Ansgar und Vera Nünning herausgegebene Sammelband Multiperspektivisches Erzählen. Zur Theorie und Geschichte der Perspektivenstruktur im englischen Roman des 18. bis 20. Jahrhunderts (Trier 2000).

41. - Vgl. etwa Lüscher, Frühling der Barbaren. a.a.O. S. 45.

42. - Vgl. Kehlmann, F. a.a.O. S. 110ff. und 196ff. 
Perspektiven, sondern kann den jeweiligen Figuren durch unterschiedliche Erzählhaltungen eine individuelle Stimme geben. Mit den Einzelperspektiven werden auch gleichzeitig persönliche Meinungen und Einstellungen kontrastierend gegenübergestellt; der Rezipient muss während des Lesevorgangs selbst eine Lösung finden, die Blickwinkel und Perspektiven bewerten und einordnen - eine narrative Strategie, die gleichzeitig Gegensätze im wirtschaftlichen Diskurs, Unsicherheiten in Zeiten der Krise, oder auch die Suche nach der Wahrheit erzählerisch spiegelt.

\section{Aufzeichnungen - wir schlafen nicht}

„Es ist so. Mich faszinieren Katastrophen“43 - Kathrin Rögglas großes Thema sind Desaster, vor allem aber die mediale Übersättigung von Natur- und Klimakatastrophen, Wirtschaftskrisen, verheerenden Unglücken oder Terroranschlägen. In verschiedenen Essays, Theaterstücken und Prosatexten beschäftigt sie sich mit deren Erscheinungsformen ebenso wie mit der ständigen Medienpräsenz; so verarbeitete sie etwa den 11. September 2001 in tagebuchartigen Aufzeichnungen, oder setzte sich in die alarmbereiten (2010) mit dem Katastrophendiskurs auseinander.

Zentral für ihre Arbeit sind jedoch die detaillierten Recherchen und akribischen Vorbereitungen, die auch ihrem Roman wir schlafen nicht vorausgingen. Dort kommen sieben exemplarische Personen in unterschiedlicher Zusammensetzung zu Wort - von ,silke mertens, key account managerin, 37 “ bis zu „herr gehringer, partner, 48 “. Sie alle geben Einblick in Arbeitsalltag, Berufsethos und Selbstbild, aber auch die Praktiken großer Konzerne, vorwiegend Banken und Finanzdienstleister.

Nicht nur bereits die dem Roman vorangestellte Auflistung der Dramatis personae erinnert an ein Theaterstïck, sondern ebenso die 33 einzelnen Kapitel, in denen die sieben Figuren abwechselnd einzeln oder in unterschiedlicher Zusammensetzung für schlaglichtartige Monologe oder kurze Gespräche auftreten, und die einem szenischen Schauspiel auf der Bühne gleichen. Aber auch formal greift Röggla auf das mehrdeutige Bild des Spiels zurück, das durch die aktuellen Wirtschaftskrisen wieder stark mit dem Turbokapitalismus wie auch dem riskanten Zocken oder der launischen Wandelbarkeit von Börsenkursen verbunden ist. So finden sich (neben der strukturgebenden Szenenfolge, sowie der durchgehenden Kleinschreibung und Konjunktivierung) auf rhetorischer Ebene sprachliche Wortspiele und innovative Neologismen:

43. - Kathrin Röggla, „Geisterstädte, Geisterfilme.“In: Dies.: besser wäre: keine . Essays und Theater, Frankfurt am Main, Fischer, 2013. S. 7. 
„mckinsey-king“44, ,minuswachstum““45, „marlboro-joblandschaft“"46, "firmenkonglomerat" 47 , „steuerszenarien“48, „schmerzabwägung“49, „alarmismus“50, „,bwl-kreisläufe“51.

Die Rhetorik eines unkommentierten, direkten und fast ungefilterten Sammelns von Aussagen und Begrifflichkeiten aus dem Bereich der Wirtschaft wurde in den vergangenen Jahren vor allem von Elfriede Jelinek zu einer literarischen Strategie ausgearbeitet, sowohl in ihrem Roman Rein Gold - paratextuell auf Richard Wagners Rheingold verweisend -, als auch in ihrem Stück Die Kontrakte des Kaufmanns. Dort baut sie in den auf der Bühne gehaltenen Monologen einen Kosmos aus floskelhaften Ausdrücken aus dem ökonomischen Diskurs auf, inhaltlich angeordnet durch Assoziationsketten: „Schauen Sie nur nach, es ist weg!, Ihr Geld ist weg!, der Kredit auch, den Sie auf Ihr Gehalt aufgenommen haben, alles futsch!, es ist lieber bei uns, aber sicher!" 52 Ein Wort führt automatisch durch ähnliche Bedeutung, Klang oder einem neuen Kontext zu einer Gedankenverknüpfung und damit zum nächsten Ausdruck. So entsteht collageartig ein Panorama, ein mehrstimmiger Chor zwischen Spekulationsverlierern und allegorisch aufgeladenen Figuren, und damit ein direkter und ungefilterter Kommunikationsvorgang (ein weiteres Spiel auf der Bühne also), das durch die Krise bedeutungslos gewordene Gemeinplätze zu neuen Sinneinheiten zusammenfügt. Zugleich werden Texte wie wir schlafen nicht oder Rein Gold aber auch fast zu Lesedramen, die noch direkter und szenischer (und dadurch noch multiperspektivischer) kontrastierend unterschiedliche Meinungen und Aussagen gegenüberstellen.

Auf der anderen Seite zeichnet sich gerade Rögglas Roman dadurch aus, dass ihm ,gespräche mit consultants, coaches, key account managerinnen, programmierern, praktikanten usw. zugrunde" 53 liegen - eine Strategie, auf die etwa auch Andres Veiel in seinem für das Staatstheater Stuttgart angefertigtem Stück Das Himbeerreich zurückgreift. Diese Fiktionalisierung von tatsächlichen Gesprächen mit Bankern, Spekulanten und Finanzdienstleistern stellt gerade bei diesen beiden Autoren eine interessante Herangehensweise an zeitaktuelle

44. - Kathrin Röggla, wir schlafen nicht, Frankfurt am Main, Fischer, 2013. S. 50.

45. - Ebd. S. 63.

46. - Ebd. S. 88.

47. - Ebd. S. 109.

48. - Ebd. S. 125.

49. - Ebd. S. 150.

50. - Ebd. S. 186.

51. - Ebd. S. 205.

52. - Jelinek, Die Kontrakte des Kaufmanns. a.a.O. S. 293.

53. - Röggla, wir schlafen nicht. a.a.O. S. 4. 
Wirtschaftskrisen dar, ermöglicht sie doch etwa durch eine authentische Sprachwahl auch Einblicke hinter die Kulissen der Finanzbranche.

\section{Frïhstück der Barbaren}

Banker, Spekulanten und Finanzdienstleister generell wurden in der Öffentlichkeit als gierig und skrupellos wahrgenommen, die in einem außer Kontrolle geratenen System ohne Regeln und Grenzen mit fremdem Geld ,zocken' konnten, nahezu ohne persönliche Konsequenzen fürchten zu müssen. Ruiniert, so der Außenblick, waren am Ende nur die Anleger, die um ihr Erspartes und die versprochenen Rendite gebracht wurden. Keine Überraschung also, dass der Spekulant während der Weltwirtschaftskrise von 2007ff. zu einer ausgesprochen polarisierenden Figur wurde, die auch von der Literatur aufgegriffen wurde.

Die ausgewählten deutschsprachigen Bearbeitungen konnten an inhaltlichen Elementen wie auch den Erzählstrategien der Prosatexte aufzeigen: Die Figur des Spekulanten gibt dem Kapitalismus angesichts einer seiner schwersten Krisen eine exemplarische Geschichte, vor allem aber auch ein Gesicht. Der Gordon Gekko des frühen 21. Jahrhunderts heißt Eric Friedland oder Bernhard Milbrandt, macht Urlaub in der tunesischen Wüste oder verkauft (!) Verluste als ,minus-wachstum'. Dabei steht aber längst nicht nur das vielleicht teils zweifelhafte Arbeitsethos des Spekulanten im Mittelpunkt der Texte, das Jonglieren mit Aktien und Positionen, das die großen Summen abstrakt zu bloßen Zahlen, zu einem Spiel mit ,Spielgeld' werden lässt.

Ohnehin, das zeigen alle Texte, lassen sich Beruf und Freizeit kaum trennen, zu sehr hat der Spekulant die ökonomischen Denkmuster verinnerlicht. So (re-)agieren die britischen Banker im tunesischen Ferienresort genauso aggressiv und destruktiv wie sie es aus der Londoner City gewohnt sind. Und nicht zufällig gehen Spekulanten wie Friedland oder Milbrandt kühl berechnend vor, wirken dabei aber auch teils panisch oder verwirrt - unter dem Einfluss von Medikamenten stehend.

Somit wird der Spekulant in seinen verschiedenen Erscheinungsformen inhaltlich zur Sozialfigur, zur archetypischen Chiffre für den Turbokapitalismus wie die zeitaktuelle Wirtschaftskrise. An ihm lässt sich der Zustand der Gesellschaft, ihrer Werte und Einstellungen reflektieren; mehr noch: Der Spekulant selbst wird, wenn er über sich, seine Zunft und das ökonomische System nachdenkt, zur Reflektionsfigur im Text. Dies zeigt sich besonders in minutiös recherchierten Werken von Röggla oder Veiel, die sich durch eine sprachliche Authentizität auszeichnen: ,die branche hat plötzlich einen schlechten ruf gehabt, das hat ihn schon so ziemlich erstaunt. es hat sie eben alle erwischt. sie alle 
sind jetzt dran"54. Doch nur selten ändert er tatsächlich sein Verhalten oder schließt mit dem System ab ${ }^{55}$, während Spekulanten von Aristide Saccard bis Eric Friedland nahezu ungestraft entkommen und sogar weiter praktizieren.

Nicht selten wird in diesem Zusammenhang die Systemfrage gestellt und der Kapitalismus als fehlerhaftes wie unkontrolliertes Monster dargestellt. Und so greift etwa die junge Geschäftsführerin Luise Tietjen in Nora Bossongs Unternehmerroman Gesellschaft mit beschränkter Haftung (2012) exemplarisch am Ende doch auf die ähnlichen Mittel frisierter Bilanzen zurück, wie ihr Vater, wobei sie sich in der stark testosteron-geprägten Welt der CEOs ohnehin kaum durchsetzen konnte. Tatsächlich ist das Thema der Generationen und ihrer unterschiedlichen Einstellungen zum Kapitalismus immer wieder motivisches Thema in den deutschsprachigen Texten (und nicht selten dabei wieder mit Bezug auf die belastete NS-Zeit, etwa bei Bossong oder Reh) - zugespitzt natürlich in der vernichtenden Generation englischer Banker im tunesischen Ferienresort.

Sicherlich lädt diese Konstellation zum plakativen Opfer-Täter-Bild und zu einfach gedachten Figurenschemata von skrupellosen Gewinnern und ahnungslosen Verlierern von Boom und Krise ein, und natürlich wurden in der Literatur der vergangenen Jahre auch solche Geschichten erzählt. Doch vielmehr scheint das aktuelle Thema der Wirtschaftskrise aber deutschsprachige Schriftsteller inspiriert und innovative Texte hervorgebracht zu haben: Der Rückgriff auf die Gattung der Novelle oder den multiperspektivischen Roman, narrative Strategien wie der polyphone Chor oder die durch Assoziationsketten strukturierte Enumeratio - ausgerechnet eine ökonomische Krise befeuert künstlerische Bearbeitungen und die Auseinandersetzung mit der gesellschaftlichen Situation.

54. - Röggla, wir schlafen nicht. a.a.O. S. 68.

55. - Wie dies etwa der Investmentbanker Roger Yount in John Lanchesters Capital tut, der mit der Weltfinanzkrise im Herbst 2008 eine regelrechte Katharsis durchläuft und auf den letzten Seiten des Romans dem System abschwört. 